\title{
gु \\ Negative refraction and backward waves in layered acoustic metamaterials
}

\author{
Johan Christensen and F. Javier García de Abajo* \\ IQFR-CSIC Serrano 119, 28006 Madrid, Spain
}

(Received 18 May 2012; published 3 July 2012)

\begin{abstract}
We investigate layered acoustic metamaterials capable of exhibiting a wide variety of wave propagation phenomena, including backward and forward waves with and without negative refraction. The metamaterials are formed by periodically perforated hard plates, which we describe analytically in the limit of small holes compared to both the period and the separation between plates. In particular, we derive expressions for the index of refraction and the transmission and reflection coefficients of finite slabs. We provide illustrative examples of near fields for all four combinations of backward and forward waves with and without negative refraction, along with dispersion diagrams that explain the observed behavior. A comprehensive study of the range of geometrical parameters in which these distinct phenomena are observed is presented as well. These metamaterials hold great promise for achieving full control of sound using simple structures, with potential application to acoustic technologies relying on subwavelength control and imaging of sound.
\end{abstract}

DOI: 10.1103/PhysRevB.86.024301

PACS number(s): 43.35.+d, 42.79.Dj, 81.05.Xj

\section{INTRODUCTION}

Sound enjoys a privileged status among the types of waves commonly encountered in everyday life and in technology (e.g., light, surface waves, etc.). It provides a natural source of information on the environment and an effective means of communication. Its propagation velocity varies orders of magnitude for different materials, and it covers a broad frequency range up to the gigahertz regime. Its interaction with interfaces is subject to Neumann-type boundary conditions (i.e., vanishing of the normal pressure gradient in hard solids), and its impedance in gas-solid boundaries can reach extremely high values. Not surprisingly, a long tradition of understanding the scattering of sound by small elements such as holes ${ }^{1-3}$ has built up, partly triggered by the goal of controlling sound propagation, ${ }^{4}$ and recently culminating in the design of acoustic metamaterials capable of molding the flow of sound down to subwavelength scales. ${ }^{5-13}$ Applications to (bio)medical ultrasonography and diagnostic imaging, ${ }^{14}$ acoustofluidic steering of microparticles and microorganisms, ${ }^{15}$ and sonochemistry ${ }^{16,17}$ have also been devised.

Among the exotic properties already realized in wave phenomena, negative refraction is appealing because of its potential to achieve perfect imaging with resolution beyond the diffraction limit (superlensing) using light waves. ${ }^{18}$ This launched a race to produce the required optical materials, which must simultaneously exhibit negative permittivity and permeability. ${ }^{19,20}$ Inspired by these results, a negative acoustic index of refraction was predicted in materials exhibiting negative effective mass density and negative bulk modulus, ${ }^{7}$ while several acoustic metamaterial designs were proposed containing resonators in the form of coated metallic spheres, ${ }^{5}$ lumped elements, ${ }^{8}$ and perforations. ${ }^{13}$ Acoustic negative refraction and backward wave propagation have also been predicted $^{21-23}$ and experimentally demonstrated ${ }^{24}$ in twodimensional sonic crystals.

The vast range of phenomena associated with sound propagion has led to applications to acoustic focusing, ${ }^{11,25-27}$ waveguiding, ${ }^{28}$ cloaking, ${ }^{29-33}$ negative refraction, ${ }^{11,34,35}$ resonant transmission through hole arrays, ${ }^{9,36-40}$ and enhanced absorption, ${ }^{41}$ which are providing a major focus of attention. In particular, cloaking has been predicted for both elastic ${ }^{29}$ and acoustic $^{30-32}$ waves, including acoustic carpets. ${ }^{32}$ Cloaking of sound has been demonstrated experimentally. ${ }^{33}$ Acoustic waves trapped at metamaterial surfaces have been investigated as well. ${ }^{42,43}$ Wave phenomena in general find their expression in sound, and even classical analogs of quantum-optics effects such as induced transparency have been realized..$^{4,45}$

The ability of perforated plates to negatively refract and focus acoustic waves has already been established. ${ }^{11}$ In a recent study, ${ }^{27}$ we proposed the use of layered metamaterials to achieve negative refraction, backward wave propagation, and subwavelength imaging. In this paper, we provide further details of the formalism used to analyze those metamaterials, along with an exhaustive search of material parameters from which we extract examples of all four combinations of negative and positive refraction with backward and forward wave propagation. This depicts an incredibly broad range of propagation phenomena in this class of metamaterials, which are reached by simply varying the wavelength in some cases, or by changing the relevant geometrical parameters in general.

\section{ANALYTICAL MODEL FOR ANISOTROPIC PERFORATED METAMATERIALS}

In this section, we elaborate an analytical model to describe acoustic metamaterials formed by periodically perforated plates, which are arranged as shown in Fig. 1(a). Holes of diameter $D$ are piercing the plates of thickness $t$. In the calculations that follow, we consider square arrays of period $d_{x}$, although the formalism is applicable to any lattice symmetry. The plates form a stack that is also periodic along the $z$ direction perpendicular to the plates with period $d_{z}$, so that the interstitial region separating consecutive plates has a width $d_{z}-t$.

For simplicity, we assume the plates to be made of a hard material embedded in a fluid. Additionally, the apertures are taken to be narrow compared to the sound wavelength $\lambda$, the distance between holes, and the separation between plates. Under these assumptions, sound only propagates in 
(a)

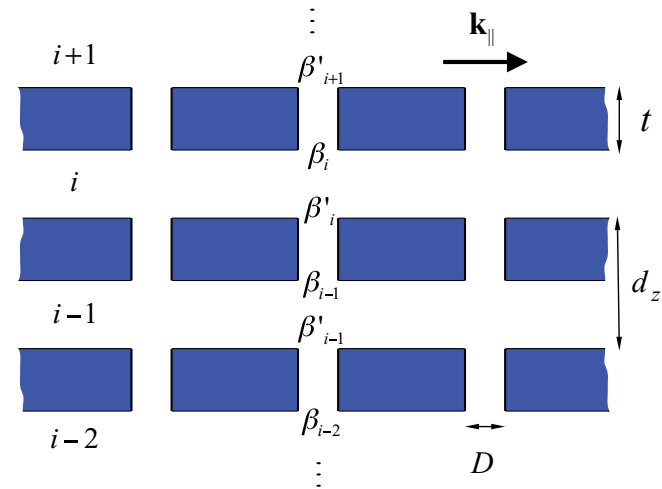

(b)

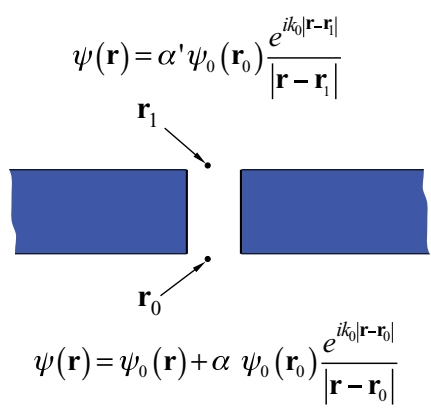

FIG. 1. (Color online) Schematic representation of a metamaterial formed by a periodic stack of perforated plates and description of the response of each of the holes. The holes form a square array of period $d_{x}$ in each plate. (a) The hole diameter $D$, the stack period $d_{z}$, and the plate thickness $t$ are shown by labels. The apertures are described by the self-consistent scattering amplitudes produced on either side of the plate. Amplitudes facing the interstitial region $i$ are denoted $\beta_{i}$ and $\beta_{i}^{\prime}$. Sound comes from the bottom and propagates toward the top. Analytical solutions are obtained here for a fixed value of the wave vector parallel to the plate, $\mathbf{k}_{\|}$. Arbitrary external sources are then represented as a sum of their different $\mathbf{k}_{\|}$components. (b) When an external pressure field $\psi_{0}(\mathbf{r})$ is applied from the lower part of a hole, monopole pressure sources are produced in response on either side of the hole, with amplitudes proportional to the incident pressure multiplied by scattering coefficients $\alpha$ and $\alpha^{\prime}$, as shown in the figure.

the fluid and the acoustic pressure must have vanishing normal derivative at the plate and hole-wall surfaces (Neumann boundary conditions).

\section{A. Bulk waves in an infinitely extended metamaterial}

It is convenient to decompose the external source of sound into Fourier components along the $x-y$ directions parallel to the plates. Then, we separately solve the propagation equations presented below for each value of the wave vector along those directions, $\mathbf{k}_{\|}$. The dependence of the monopole amplitudes on the position of the holes $\mathbf{R}_{j}$ along the planar arrays is given by $\exp \left(i \mathbf{k}_{\|} \cdot \mathbf{R}_{j}\right)$. The amplitudes of the upper and lower apertures facing each interstitial region $i$ are denoted $\beta_{i} \exp \left(i \mathbf{k}_{\|} \cdot \mathbf{R}_{j}\right)$ and $\beta_{i}^{\prime} \exp \left(i \mathbf{k}_{\|} \cdot \mathbf{R}_{j}\right)$, respectively [see Fig. 1(a)].

Assuming that the apertures are small compared to both their separation and the sound wavelength, the response of each hole is given by the amplitudes of the scattering monopoles that it produces on its upper and lower parts. These amplitudes depend on the incident pressure acting on the hole, as illustrated in Fig. 1(b). Analytical expressions for the monopole scattering coefficients $\alpha$ and $\alpha^{\prime}$ [see Fig. 1(b)] for a hole infiltrated with a fluid are derived in detail in the supplemental material of Ref. 27:

$$
\begin{aligned}
& g^{+}=\frac{1}{\alpha+\alpha^{\prime}}=\frac{-8}{k_{0} D^{2}}\left[2 i I-\eta \cot \left(k_{1} t / 2\right)\right], \\
& g^{-}=\frac{1}{\alpha-\alpha^{\prime}}=\frac{-8}{k_{0} D^{2}}\left[2 i I+\eta \tan \left(k_{1} t / 2\right)\right],
\end{aligned}
$$

where $k_{0}=2 \pi / \lambda$ is the sound wave vector in the fluid outside the hole, $k_{1}$ is the wave vector in the fluid inside the hole, $\eta=\rho_{1} k_{0} / \rho_{0} k_{1}$ is the acoustic impedance involving the density inside $\left(\rho_{1}\right)$ and outside $\left(\rho_{0}\right)$ the hole, and

$$
I \approx\left(k_{0}^{2} D^{2} / 16\right)\left(1-32 i / 3 \pi k_{0} D\right) .
$$

These expressions, which are valid under the current assumption $k_{0} D \ll 1$, satisfy the optical theorem ${ }^{27}$

$$
\operatorname{Im}\left\{g^{ \pm}\right\} \leqslant-k_{0},
$$

where the equal stands for nonabsorbing holes.

Now, using a procedure similar to a previous analysis for periodic particle arrays, ${ }^{46}$ we can write the following selfconsistent relation for the scattering amplitudes of a given hole, which are produced in response to the sum of monopole contributions from all other holes in the two interstitial regions that this hole communicates:

$$
\begin{aligned}
\beta_{i}^{\prime} & =\alpha\left(G^{\prime} \beta_{i}+G \beta_{i}^{\prime}\right)+\alpha^{\prime}\left(G^{\prime} \beta_{i-1}^{\prime}+G \beta_{i-1}\right), \\
\beta_{i-1} & =\alpha^{\prime}\left(G^{\prime} \beta_{i}+G \beta_{i}^{\prime}\right)+\alpha\left(G^{\prime} \beta_{i-1}^{\prime}+G \beta_{i-1}\right),
\end{aligned}
$$

where $G$ describes the interaction with apertures in the same plate as the given hole and $G^{\prime}$ represents the contribution of holes in the contiguous plates, which are placed an interstitial region apart. For example, the first of these equations describes the scattering amplitude $\beta_{i}^{\prime}$ of the lower apertures in the interstitial region $i$, which is the sum of two contributions: the scattering of the field produced by holes in region $i$, via the scattering coefficient $\alpha$, and the scattering of the field coming from apertures in region $i-1$, via $\alpha^{\prime}$. The first of these contributions involves both a sum over openings in the same plate as the given hole (terms proportional to $G$ ) and a sum over lattice sites in the plate immediately above it [terms in $G^{\prime}$, see Fig. 1(a)]. The rest of the terms admit a similarly intuitive interpretation.

The lattice sums are easily constructed from the direct monopoles induced at the hole apertures and the subsequent infinite series of identical monopole mirror images upon reflection on the plate boundaries. This allows us to write

$$
\begin{aligned}
G & =\sum_{j} \sum_{n=-\infty}^{\infty} \frac{\mathrm{e}^{i k_{0} \sqrt{R_{j}^{2}+4 n^{2}\left(d_{z}-t\right)^{2}}}}{\sqrt{R_{j}^{2}+4 n^{2}\left(d_{z}-t\right)^{2}}} \mathrm{e}^{i \mathbf{k}_{\|} \cdot \mathbf{R}_{j}}, \\
G^{\prime} & =\sum_{j} \sum_{n=-\infty}^{\infty} \frac{\mathrm{e}^{i k_{0} \sqrt{R_{j}^{2}+(2 n+1)^{2}\left(d_{z}-t\right)^{2}}}}{\sqrt{R_{j}^{2}+(2 n+1)^{2}\left(d_{z}-t\right)^{2}}} \mathrm{e}^{i \mathbf{k}_{\|} \cdot \mathbf{R}_{j}},
\end{aligned}
$$

where $\mathbf{R}_{j}$ runs over the 2 D lattice of holes and the sum in $G$ is such that either $\mathbf{R}_{j} \neq 0$ or $n \neq 0$. Ewald's method is ideally suited for a fast evaluation of these expressions, as shown 
explicitly in the supplemental material of Ref. 27, where the important properties

$$
\begin{aligned}
\operatorname{Im}\{G\} & =-k_{0}, \\
\operatorname{Im}\left\{G^{\prime}\right\} & =0
\end{aligned}
$$

are also derived in detail.

It is convenient to write Eqs. (3) in matrix notation as

$$
\left[\begin{array}{l}
\beta_{i}^{\prime} \\
\beta_{i}
\end{array}\right]=T\left[\begin{array}{l}
\beta_{i-1}^{\prime} \\
\beta_{i-1}
\end{array}\right]
$$

where

$$
\begin{aligned}
T & =\left[\begin{array}{ll}
a & b \\
-b & d
\end{array}\right], \quad a=\frac{\alpha^{\prime 2}-\alpha^{2}}{\alpha^{\prime}} G^{\prime}, \\
b & =\frac{\alpha^{\prime 2}-\alpha^{2}}{\alpha^{\prime}} G+\frac{\alpha}{\alpha^{\prime}}, \quad d=\frac{(1-\alpha G)^{2}-\alpha^{\prime 2} G^{2}}{\alpha^{\prime} G^{\prime}} .
\end{aligned}
$$

Now, we define the component of the wave vector along the plates normal $k_{z}$ for waves propagating in the metamaterial in terms of the eigenvalues of $\mathrm{T}$,

$$
\mathrm{e}^{ \pm i k_{z} d_{z}}=\xi \pm \sqrt{\xi^{2}-1}
$$

where

$$
\xi=\frac{1}{\left(g^{-}-g^{+}\right) G^{\prime}}\left[\left(G-g^{+}\right)\left(G-g^{-}\right)-G^{\prime 2}\right] .
$$

In writing Eq. (6) we have used the property that $T$ has unit determinant (i.e., the product of its two eigenvalues must be equal to 1). Using the optical theorem [Eq. (2)] for nonabsorbing plates $\left(\operatorname{Im}\left\{g^{ \pm}\right\}=-k_{0}\right)$ together with Eqs. (4), we find that

$\xi=\frac{1}{\operatorname{Re}\left\{g^{-}-g^{+}\right\} G^{\prime}}\left[\operatorname{Re}\left\{G-g^{+}\right\} \operatorname{Re}\left\{G-g^{-}\right\}-G^{\prime 2}\right]$

is a real number. And from Eq. (6), we conclude that $k_{z}$ is imaginary for $|\xi|>1$, implying that wave propagation is forbidden in the metamaterial. In contrast, propagating modes exist under the condition $|\xi| \leqslant 1$, which leads to real wave vectors

$$
k_{z}= \pm \frac{1}{d_{z}} \cos ^{-1} \xi,
$$

where the \pm sign is determined by the physical condition $\operatorname{Im}\left\{k_{z}\right\}>0$ (see below) and we take $0 \leqslant \cos ^{-1} \xi \leqslant \pi$.

In our retarded response formalism, waves propagate in the direction of positive $\operatorname{Im}\left\{k_{z}\right\}$ as a requirement of energy conservation. Then, backward wave propagation is signaled by the condition that $\operatorname{Re}\left\{k_{z}\right\}$ and $\operatorname{Im}\left\{k_{z}\right\}$ have opposite signs. Here, we introduce absorption inside the holes through a small positive imaginary part in $k_{1}=k_{1}^{r}+i k_{1}^{i}$ [see Eqs. (1)], so that $\operatorname{Im}\left\{g^{ \pm}\right\} \approx-k_{0}-k_{1}^{i} \zeta_{ \pm}$, where $\zeta_{ \pm}=-\partial g^{ \pm} / \partial k_{1}$. More precisely,

$$
\begin{aligned}
\zeta^{+} & =\frac{4 \eta t}{k_{0} D^{2}} \frac{1}{\cos ^{2}\left(k_{1} t / 2\right)}, \\
\zeta^{-} & =\frac{4 \eta t}{k_{0} D^{2}} \frac{1}{\sin ^{2}\left(k_{1} t / 2\right)}
\end{aligned}
$$

Now, Taylor expanding Eq. (7), we find that Eq. (8) must be supplemented by a small imaginary part given by

$$
\begin{aligned}
\operatorname{Im}\{\xi\}= & \frac{1}{\left[\left(\operatorname{Re}\left\{g^{-}-g^{+}\right\}\right)^{2}+\left(\zeta_{+}-\zeta_{-}\right)^{2}\right] G^{\prime}} \\
& \times\left[\left(\operatorname{Re}\left\{G-g^{+}\right\}\right)^{2} \zeta_{-}-\left(\operatorname{Re}\left\{G-g^{-}\right\}\right)^{2} \zeta_{+}\right. \\
& \left.+\left(G^{\prime 2}+\zeta_{+} \zeta_{-}\right)\left(\zeta_{+}-\zeta_{-}\right)\right] .
\end{aligned}
$$

Finally, we find from Eq. (6) that $\operatorname{Re}\left\{k_{z}\right\}$ and $\operatorname{Im}\left\{k_{z}\right\}$ have different signs (the condition for backward wave propagation) if and only if $\operatorname{Im}\{\xi\}>0$, in which case the - sign has to be chosen in Eq. (9) in order to have $\operatorname{Im}\left\{k_{z}\right\}>0$. Otherwise, we have forward waves and the + sign must be used.

\section{B. Reflection and transmission coefficients of finite metamaterial films}

For a finite metamaterial stack in which sound is incident and reflected in region $i=0$ and transmitted to the top in region $i=N$, we can proceed in a way similar to the derivation of Eqs. (3) and (5) for the upper and lower plates. We find

$$
\begin{aligned}
& {\left[\begin{array}{c}
\beta_{1}^{\prime} \\
\beta_{1}
\end{array}\right]=T_{0}\left[\begin{array}{c}
2 \psi^{\mathrm{inc}} / G^{\prime} \\
\beta_{0}
\end{array}\right],} \\
& {\left[\begin{array}{c}
\beta_{N}^{\prime} \\
0
\end{array}\right]=T_{N}\left[\begin{array}{l}
\beta_{N-1}^{\prime} \\
\beta_{N-1}
\end{array}\right],}
\end{aligned}
$$

where the matrices

$$
T_{0}=\left[\begin{array}{ll}
a & b^{\prime} \\
-b & d^{\prime}
\end{array}\right], \quad T_{N}=\left[\begin{array}{ll}
a & b \\
-b^{\prime} & d^{\prime}
\end{array}\right]
$$

have unit determinant, and

$$
\begin{aligned}
b^{\prime} & =\frac{\alpha^{\prime 2}-\alpha^{2}}{\alpha^{\prime}} G_{0}+\frac{\alpha}{\alpha^{\prime}}, \\
d^{\prime} & =\frac{(1-\alpha G)\left(1-\alpha G_{0}\right)-\alpha^{\prime 2} G G_{0}}{\alpha^{\prime} G^{\prime}} .
\end{aligned}
$$

The factor of 2 in Eq. (10a) originates in the wave directly reflected by the lower plate in the absence of the holes. Here, $G_{0}$ is the sum over hole positions in the top and bottom planar interfaces of the overall structure,

$$
G_{0}=\sum_{\mathbf{R}_{j} \neq 0} \frac{\mathrm{e}^{i k_{0} R_{j}}}{R_{j}} \mathrm{e}^{i \mathbf{k}_{\|} \cdot \mathbf{R}_{j}} .
$$

We evaluate $G_{0}$ following the methods elaborated by Kambe. ${ }^{47}$ Its imaginary part below the diffraction threshold admits the analytical expressions ${ }^{38} \operatorname{Im}\left\{G_{0}\right\}=-k_{0}$ outside the sound cone and $\operatorname{Im}\left\{G_{0}\right\}=-k_{0}+2 \pi / k_{\perp} A$ inside it, where $k_{\perp}=$ $\sqrt{k_{0}^{2}-k_{\|}^{2}+i 0^{+}}$is the normal wave vector outside the metamaterial and $A$ is the unit cell area of the hole arrays.

Using Eqs. (5) and (10), the near- and far-side amplitudes $\beta_{0}$ and $\beta_{N}^{\prime}$ are related by

$$
\left[\begin{array}{c}
\beta_{N}^{\prime} \\
0
\end{array}\right]=T_{N} T^{N-2} T_{0}\left[\begin{array}{c}
2 \psi^{\mathrm{inc}} / G^{\prime} \\
\beta_{0}
\end{array}\right] .
$$




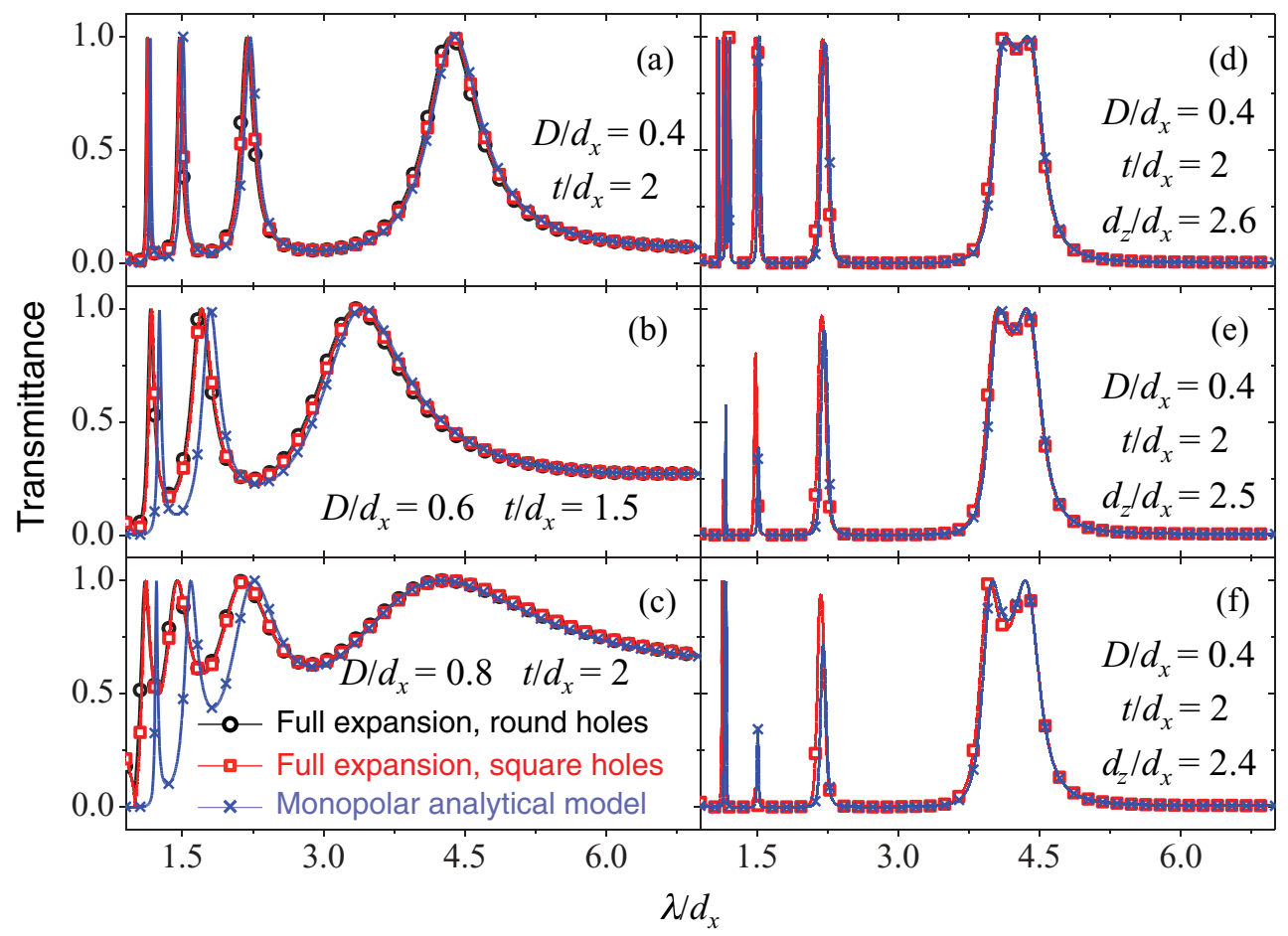

FIG. 2. (Color online) Acoustic normal-incidence transmittance through one (a)-(c) and two (d)-(e) perforated plates arranged as in Fig. 1(a), with geometrical parameters as shown by labels. The results of our monopole analytical formalism (blue curves and crosses) are compared with full-wave expansions of the pressure field, both for round holes (black curves and circles) and square holes of the same area (red curves and squares). All lengths are normalized to the period of the square hole array $d_{x}$. The fluid is the same inside and outside the holes.

Incidentally, it is useful to change to a basis set in which $T$ is diagonal, which allows us to readily evaluate $T^{N-2}$ as

$$
\begin{aligned}
T^{N-2}= & \frac{-1}{2 b \sin \left(k_{z} d_{z}\right)}\left[\begin{array}{cc}
-b & -b \\
a-\mathrm{e}^{i k_{z} d_{z}} & a-\mathrm{e}^{-i k_{z} d_{z}}
\end{array}\right] \\
& \times\left[\begin{array}{cc}
\mathrm{e}^{i k_{z} d_{z}(N-2)} & 0 \\
0 & \mathrm{e}^{-i k_{z} d_{z}(N-2)}
\end{array}\right]\left[\begin{array}{cc}
a-\mathrm{e}^{-i k_{z} d_{z}} & b \\
-a+\mathrm{e}^{i k_{z} d_{z}} & -d
\end{array}\right] .
\end{aligned}
$$

Now, introducing the explicit notation for the elements of the transmission matrix

$$
T_{N} T^{N-2} T_{0}=\left[\begin{array}{ll}
\tau_{11} & \tau_{12} \\
\tau_{21} & \tau_{22}
\end{array}\right],
$$

we find

$$
\begin{aligned}
\beta_{0} / \psi^{\text {inc }} & =-2 \tau_{21} / \tau_{22} G^{\prime}, \\
\beta_{N}^{\prime} / \psi^{\text {inc }} & =2\left(\tau_{11} \tau_{22}-\tau_{12} \tau_{21}\right) / \tau_{22} G^{\prime} .
\end{aligned}
$$
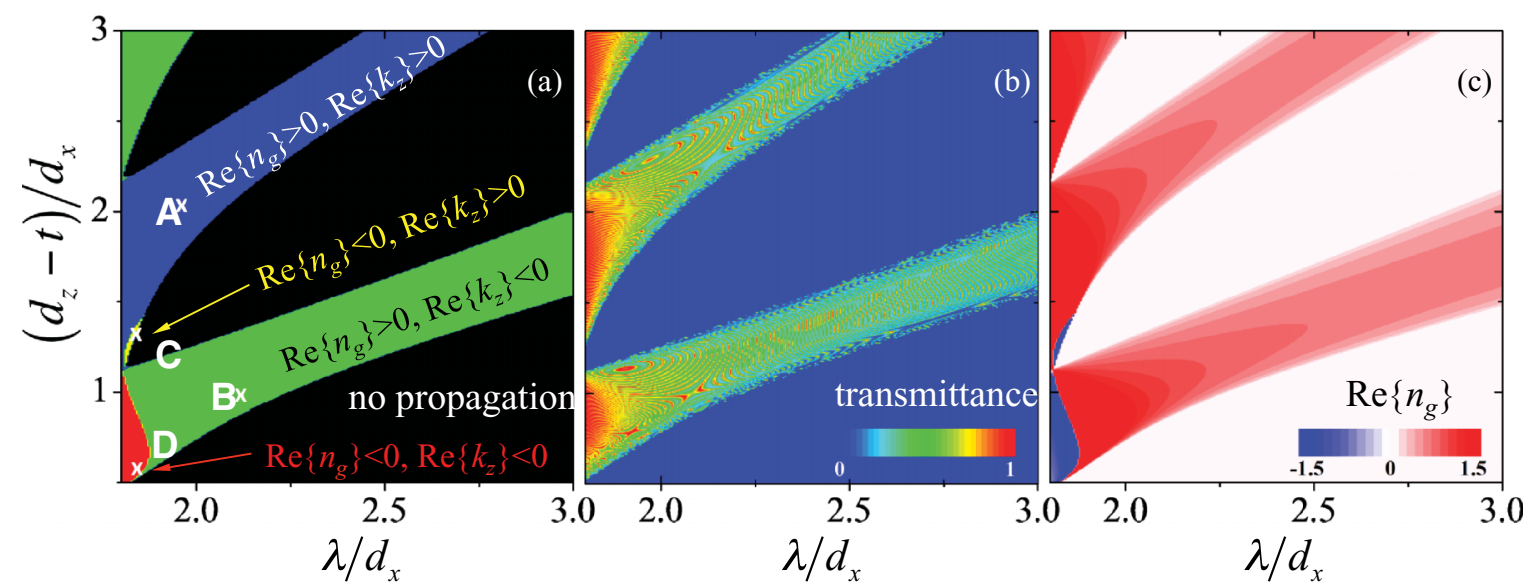

FIG. 3. (Color online) (a) Regimes of wave propagation inside a metamaterial as a function of wavelength and hole array spacing (see Fig. 1) for $D / d_{x}=0.4, t / d_{x}=0.5, \eta=0.6$ [see Eq. (1)], and $k_{\|}=\pi / \lambda$ (30 angle of incidence). All lengths are normalized to the period of the square hole array $d_{x}$. The wave vector inside the holes is the same as outside $\left(k_{1}=k_{0}\right)$. Regions of no propagation correspond to $\operatorname{Im}\left\{k_{z}\right\}>0.02 k_{0}$. (b) Transmittance through a metamaterial slab consisting of $N=250$ perforated plates. (c) Real part of the index of refraction $n_{g}$. 
These expressions are valid for metamaterial slabs containing $N \geqslant 2$ perforated plates. For $N=1$, one simply has $\beta_{0}=$ $\left(g_{+}-G_{0}\right)^{-1}+\left(g_{-}-G_{0}\right)^{-1}$ and $\beta_{1}^{\prime}=\left(g_{+}-G_{0}\right)^{-1}-\left(g_{-}-\right.$ $\left.G_{0}\right)^{-1}$. Finally, summing over the monopole contributions to the far-field pressure coming from holes in the near and far sides of the structure, the reflection and transmission coefficients reduce to

$$
r_{\mathbf{k}_{\|}}=1+\frac{2 \pi i\left(\beta_{0} / \psi^{\text {inc }}\right)}{A k_{\perp}}, \quad t_{\mathbf{k}_{\|}}=\frac{2 \pi i\left(\beta_{N}^{\prime} / \psi^{\text {inc }}\right)}{A k_{\perp}} .
$$

In the absence of diffraction for nonabsorbing materials, we have $\left|r_{\mathbf{k}_{\|}}\right|^{2}+\left|t_{\mathbf{k}_{\|}}\right|^{2}=1$.

\section{Accuracy of the monopole model}

We test the accuracy of our monopole analytical approach by comparing results for one and two perforated plates obtained with a complete full-mode expansion of the pressure field, available for square ${ }^{48}$ and circular ${ }^{39}$ holes. We are considering circular holes in the analytical approach, so we take the same hole areas in both calculations, as the actual hole shape is expected to play a minor role for small apertures. Figure 2 shows the resulting transmittance for reasonable choices of geometrical parameters. The transmittance is represented as a function of the wavelength relative to the hole spacing, $\lambda / d_{x}$. These are the basic building blocks of our metamaterials, so it is important to show that they are well described within the analytical formalism. We conclude from Fig. 2 that the results of rigorous calculations agree qualitatively well with our monopole-based model, and even the quantitative agreement is rather satisfactory down to relatively low values of $\lambda / d_{x}$.

\section{WAVE PROPAGATION REGIMES}

We are now ready to explore the exotic wave propagation properties of our layered perforated metamaterials. In particular, we tune the parameters of the structure to exhibit negative and positive refraction with backward or forward wave propagation. For this purpose, we must be careful when defining the phase and group velocities $\mathbf{v}_{p}$ and $\mathbf{v}_{g}$, as well as the group refraction index $n_{g}$.

The phase velocity describes the evolution of equal-phase planes in a plane wave mode and can be written in terms of the wave vector inside the metamaterial $\mathbf{k}=\mathbf{k}_{\|}+k_{z} \hat{\mathbf{z}}$ and the frequency $\omega$ as $\mathbf{v}_{p}=\omega \mathbf{k} /|\mathbf{k}|^{2}$. In contrast, the group velocity describes the direction and speed of energy propagation inside the material and is associated with the condition of stationary phase in $\exp (i \mathbf{k} \cdot \mathbf{r})$, which leads to the well-known expression $\mathbf{v}_{g}=\nabla_{\mathbf{k}} \omega$, indicating that the group velocity is normal to the equifrequency surfaces in wave-vector space. Since we have $k_{z}$ expressed in terms of $\mathbf{k}_{\|}$and $\omega$ in Eq. (9), instead of $\omega$ in terms of $\mathbf{k}_{\|}$and $k_{z}$, we need to transform $\nabla_{\mathbf{k}} \omega$ into the equivalent expression

$$
\mathbf{v}_{g}=\frac{1}{\partial k_{z} / \partial \omega}\left(-\nabla_{\mathbf{k}_{\|}} k_{z}+\hat{\mathbf{z}}\right)
$$

For $\mathbf{k}_{\|}=k_{\|} \hat{\mathbf{x}}$ along a high-symmetry direction $\hat{\mathbf{x}}$ of the planar hole arrays, we find $\nabla_{\mathbf{k}_{\|}} k_{z}=\left(\partial k_{z} / \partial k_{\|}\right) \hat{\mathbf{x}}$. It is also convenient to define the group index of refraction $n_{g}$ from Snell's law,
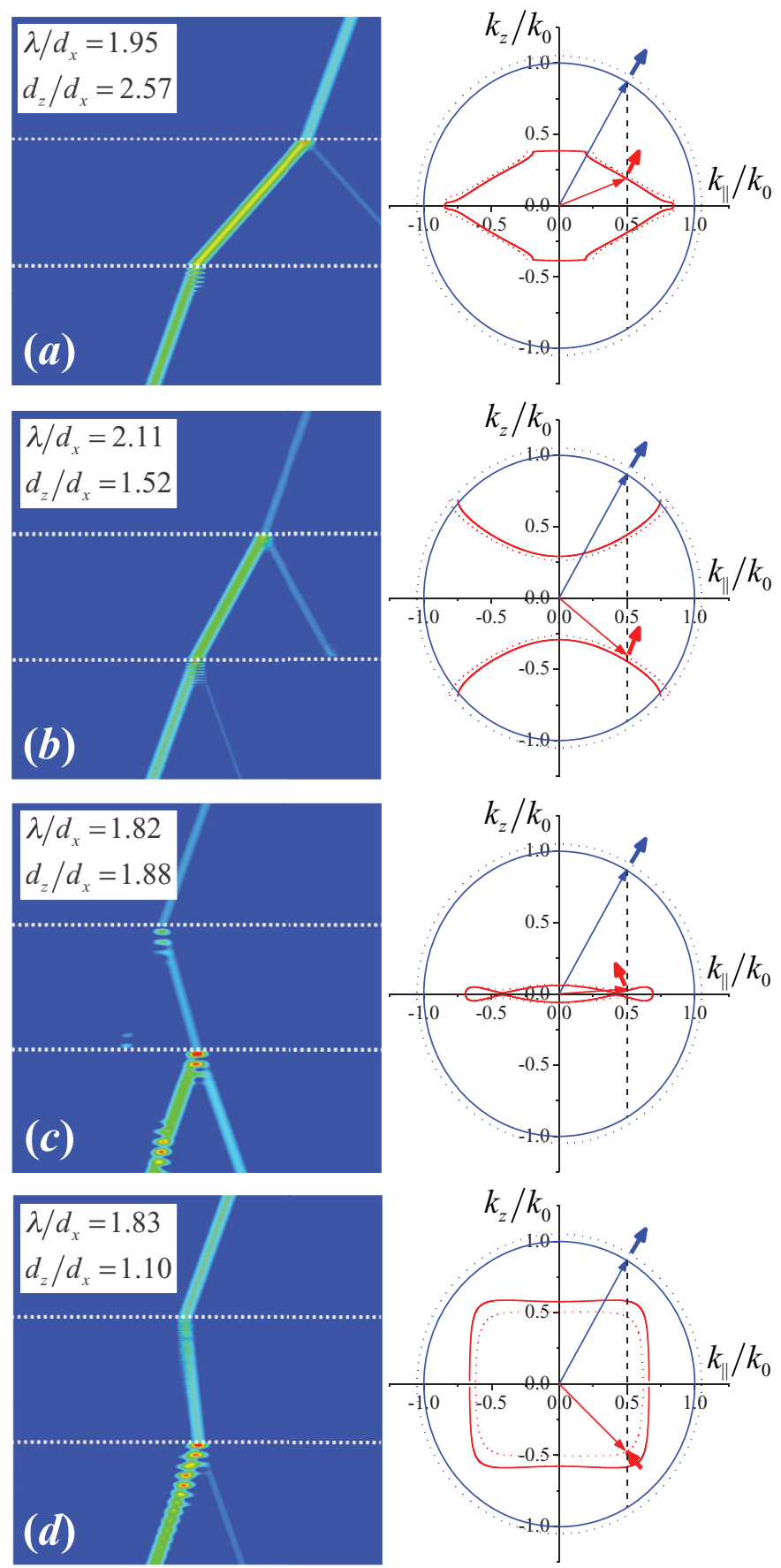

FIG. 4. (Color online) Left: Near-field pressure intensity plots for all four combinations of backward and forward waves with and without negative refraction. The conditions for plots (a)-(d) correspond to the crosses A-D of Fig. 3(a), with $30^{\circ}$ incidence angle. The broken lines indicate the slab interfaces. Right: Equifrequency curves for sound propagation in free space (blue curves) and in the metamaterials (red curves), for the same geometrical parameters and wavelengths as in the left plots. The group (phase) velocity is shown as thick (thin) arrows. Equifrequency curves for slightly larger frequency are added as broken curves to illustrate the direction of the group velocity. The parallel component of the phase velocity is conserved across the interface. The transmission group velocity must be oriented towards positive $z$ 's, and this determines the sign of the phase velocity once inside the metamaterial. 
which yields

$$
n_{g}=-\frac{\left(k_{\|} / k_{0}\right)}{\partial k_{z} / \partial k_{\|}} \sqrt{1+\left(\partial k_{z} / \partial k_{\|}\right)^{2}} .
$$

The value of $k_{\|}$is determined by the direction of the incident wave vector in the near side of the metamaterial. Assuming that the external medium is a conventional fluid, the incident wave moves toward the right for $k_{\|}>0$. However, the group velocity inside the metamaterial can be directed either toward the left (negative refraction) or toward the right (positive refraction), depending whether the sign of $\partial k_{z} / \partial k_{\|}$is positive or negative, respectively. Additionally, positive (negative) refraction is signaled by $\operatorname{Re}\left\{n_{g}\right\}>0\left(\operatorname{Re}\left\{n_{g}\right\}<0\right)$, according to Eq. (11).

In order to distinguish between different wave propagation regimes, we separate in Fig. 3(a) different regions according to the signs of $\operatorname{Re}\left\{k_{z}\right\}$ and $\operatorname{Re}\left\{n_{g}\right\}$ [see Eqs. (9) and (11)] for a wide range of wavelengths and plate separations. We consider holes of diameter $D / d_{x}=0.4$, thickness $t / d_{x}=0.5$, and impedance $\eta=0.6$ [see Eq. (1)], and we fix the parallel wave-vector $k_{\|}=0.5 k_{0}$. Regions for all four combinations of positive and negative refraction $\left(\operatorname{Re}\left\{n_{g}\right\}>0\right.$ and $\left.\operatorname{Re}\left\{n_{g}\right\}<0\right)$ with forward and backward propagation $\left(\operatorname{Re}\left\{k_{z}\right\}>0\right.$ and $\left.\operatorname{Re}\left\{k_{z}\right\}<0\right)$ are identified. We further show in Fig. 3(b) the transmittance associated with the regimes of wave propagation for a finite metamaterial stack consisting of $N=250$ perforated plates. The transmittance displays large values for the regimes of unusual wave propagation from Fig. 3(a), mainly at small wavelengths, while the index of refraction takes moderate values, as shown in Fig. 3(c).

We illustrate in Fig. 4 these four different propagation regimes through near-field pressure maps for an incident Gaussian beam at the specific values of $d_{z}-t$ and $\lambda$ indicated by crosses in Fig. 3(a). Additionally, we show equifrequency curves (right plots of Fig. 4, solid curves) obtained by plotting $k_{z}$ [Eq. (9)] as a function of $k_{\|}$. For free-space wave propagation (blue curves), the group velocity (thick arrows) coincides with the phase velocity (thin arrows, not to scale). The four scenarios represented in Fig. 4 clearly illustrate each of the four different propagation regimes. As pointed out above, the group velocity (and hence the direction of energy flow) is normal to the equifrequency curves and oriented toward the direction of increasing frequency (dotted equifrequency curves are shown at slightly higher frequency).

In contrast to Fig. 4(a), which resembles an anisotropic medium of elliptical equifrequency curves, we find in Fig. 4(b) a hyperbolic metamaterial behavior that gives rise to backward wave propagation with positive refraction, characterized by a different direction of phase propagation and intensity flux with respect to the interface normal. Varying the wavelength and the $d_{z} / d_{x}$ ratio slightly, as indicated and calculated in Figs. 4(c) and 4(d), we predict that the incident Gaussian beam undergoes negative refraction. Although the equifrequency curve of Fig. 4(c) again displays signs of anisotropy, we clearly see that the free-space and metamaterial group velocities are oriented towards different directions with respect to the interface normal, which is a clear indication of negative refraction, as confirmed by the pressure field map. Additionally, a broadangle negative-index behavior is predicted by the right plot of Fig. 4(d), leading to group and phase velocities with opposite sign in their $z$ components. Remarkably, this vast range of wave phenomena occurs within the same type of metamaterial structure, in which only the ratios $d_{z} / d_{x}$ and $\lambda / d_{x}$ are changed.

\section{CONCLUSIONS}

We have theoretically analyzed layered acoustic metamaterials based upon perforated plates and shown that these structures sustain backward and forward propagating waves with and without negative refraction. We have derived analytical expressions for the acoustic transmission and reflection coefficients of metamaterial slabs and explored different geometrical parameters to show that all four combinations of propagation regimes are reachable by suitably choosing those parameters. A wide variety of material properties can be obtained by changing the separation and size of the holes, or even by scanning the wavelength for a fixed metamaterial configuration, and thus, we foresee that these layered structures can find important applications to the field of subwavelength acoustic imaging, as well as to focusing ${ }^{26,27}$ and guiding ${ }^{34}$ of sound, adding up to recent alternative designs with extraordinary acoustic properties. ${ }^{35}$

\section{ACKNOWLEDGMENTS}

This work has been supported by the Spanish MICINN (MAT2010-14885 and Consolider NanoLight.es) and the European Commission (FP7-ICT-2009-4-248909-LIMA and FP7-ICT-2009-4-248855-N4E). J. C. gratefully acknowledges financial support from the Carlsberg Foundation under Contract No. MetaSound 2011-01-0099.

\footnotetext{
*Corresponding author: J.G.deAbajo@csic.es

${ }^{1}$ C. J. Bouwkamp, Theoretische En Numerieke Behandeling van de Buiging Door Een Ronde Opening, Dissertation (University of Groningen, Groningen, 1940).

${ }^{2}$ R. D. Spence, J. Acoust. Soc. Am. 20, 380 (1948).

${ }^{3}$ G. P. Wilson and W. W. Soroka, J. Acoust. Soc. Am. 37, 286 (1965).

${ }^{4}$ G. W. Stewart, Phys. Rev. 20, 528 (1922).

${ }^{5}$ Z. Y. Liu, X. X. Zhang, Y. W. Mao, Y. Y. Zhu, Z. Y. Yang, C. T. C. CT, and P. Sheng, Science 289, 1734 (2000).

${ }^{6}$ D. R. Smith and D. Schurig, Phys. Rev. Lett. 90, 077405 (2003).
}

${ }^{7}$ J. Li and C. T. Chan, Phys. Rev. E 70, 055602(R) (2004).

${ }^{8}$ N. Fang, D. Xi, J. Xu, M. Ambati, W. Srituravanich, and X. Zhang, Nat. Mater. 5, 452 (2006)

${ }^{9}$ H. Estrada, P. Candelas, A. Uris, F. Belmar, F. J. García de Abajo, and F. Meseguer, Phys. Rev. Lett. 101, 084302 (2008).

${ }^{10}$ S. Zhang, L. Yin, and N. Fang, Phys. Rev. Lett. 102, 194301 (2009).

${ }^{11}$ M. Farhat, S. Guenneau, S. Enoch, A. B. Movchan, and G. G. Petursson, Appl. Phys. Lett. 96, 081909 (2010).

${ }^{12}$ S. H. Lee, C. M. Park, Y. M. Seo, Z. G. Wang, and C. K. Kim, Phys. Rev. Lett. 104, 054301 (2010). 
${ }^{13}$ J. Zhu, J. Christensen, J. Jung, L. Martín-Moreno, X. Yin, L. Fok, X. Zhang, and F. J. García-Vidal, Nat. Phys. 7, 52 (2011).

${ }^{14}$ M. Sackmann, M. Delius, T. Sauerbrucht, J. Holl, W. Weber, E. Ippish, U. Hagelauer, O. Wess, W. Hepp, W. Brendel et al., N. Engl. J. Med. 318, 393 (1988).

${ }^{15} \mathrm{H}$. Bruus, Theoretical Microfluidics, Oxford Master Series in Physics (Oxford University Press, Oxford, 2008).

${ }^{16}$ K. S. Suslick, Science 247, 1439 (1990).

${ }^{17}$ Y. T. Didenko, W. B. McNamara, and K. S. Suslick, Phys. Rev. Lett. 84, 777 (2000).

${ }^{18}$ J. B. Pendry, Phys. Rev. Lett. 85, 3966 (2000).

${ }^{19}$ J. B. Pendry, A. J. Holden, D. J. Robbins, and W. J. Stewart, IEEE Trans. Microw. Theory Tech. 47, 2075 (1999).

${ }^{20}$ J. Valentine, S. Zhang, T. Zentgraf, E. Ulin-Avila, D. A. Genov, G. Bartal, and X. Zhang, Nature 455, 376 (2008).

${ }^{21}$ X. Zhang and Z. Liu, Appl. Phys. Lett. 85, 341 (2004).

${ }^{22}$ A. C. Hladky-Hennion, J. Vasseur, B. Dubus, B. DjafariRouhani, D. Ekeom, and B. Morvan, J. Appl. Phys. 104, 064906 (2008).

${ }^{23}$ A. Sukhovich, L. Jing, and J. H. Page, Phys. Rev. B 77, 014301 (2008).

${ }^{24}$ L. Feng, X. P. Liu, M. H. Lu, Y. B. Chen, Y. F. Chen, Y. W. Mao, J. Zi, Y. Y. Zhu, S. N. Zhu, and N. B. Ming, Phys. Rev. Lett. 96, 014301 (2006).

${ }^{25}$ S. Guenneau, A. Movchan, G. Pétursson, and S. A. Ramakrishna, New J. Phys. 9, 399 (2007).

${ }^{26}$ Z. He, F. Cai, Y. Ding, and Z. Liu, Appl. Phys. Lett. 93, 233503 (2008).

${ }^{27}$ J. Christensen and F. J. García de Abajo, Phys. Rev. Lett. 108, 124301 (2012).

${ }^{28}$ J. Christensen, P. A. Huidobro, L. Martín-Moreno, and F. J. GarcíaVidal, Appl. Phys. Lett. 93, 083502 (2008).

${ }^{29}$ G. W. Milton, M. Briane, and J. R. Willis, New J. Phys. 8, 248 (2006).
${ }^{30}$ H. Chena and C. T. Chan, Appl. Phys. Lett. 91, 183518 (2007).

${ }^{31}$ S. A. Cummer, B. I. Popa, D. Schurig, D. R. Smith, J. Pendry, M. Rahm, and A. Starr, Phys. Rev. Lett. 100, 024301 (2008).

${ }^{32}$ G. Dupont, M. Farhat, A. Diatta, S. Guenneau, and S. Enoch, Wave Motion 48, 483 (2011).

${ }^{33}$ B. I. Popa, L. Zigoneanu, and S. A. Cummer, Phys. Rev. Lett. 106, 253901 (2011).

${ }^{34}$ L. Fok and X. Zhang, Phys. Rev. B 83, 214304 (2011).

${ }^{35}$ Z. Liang and J. Li, Phys. Rev. Lett. 108, 114301 (2012).

${ }^{36}$ B. Hou, J. Mei, M. Ke, Z. Liu, J. Shi, and W. Wen, J. Appl. Phys. 104, 014909 (2008).

${ }^{37}$ H. Estrada, F. J. García de Abajo, P. Candelas, A. Uris, F. Belmar, and F. Meseguer, Phys. Rev. Lett. 102, 144301 (2009).

${ }^{38}$ F. J. García de Abajo, H. Estrada, and F. Meseguer, New J. Phys. 11, 093013 (2009).

${ }^{39}$ H. Estrada, P. Candelas, A. Uris, F. Belmar, F. Meseguer, and F. J. García de Abajo, Wave, Motion 48, 235 (2011).

${ }^{40}$ J. Christensen, L. Martín-Moreno, and F. J. García-Vidal, Phys. Rev. Lett. 101, 014301 (2008).

${ }^{41}$ J. Mei, G. Ma, M. Yang, Z. Yang, W. Wen, and P. Sheng, Nat. Commun. 3, 756 (2012).

${ }^{42}$ M. Ke, Z. He, S. Peng, Z. Liu, J. Shi, W. Wen, and P. Sheng, Phys. Rev. Lett. 99, 044301 (2007).

${ }^{43}$ H. Estrada, P. Candelas, F. Belmar, A. Uris, F. J. García de Abajo, and F. Meseguer, Phys. Rev. B 85, 174301 (2012).

${ }^{44}$ F. Liu, M. Ke, A. Zhang, W. Wen, J. Shi, Z. Liu, and P. Sheng, Phys. Rev. B 82, 026601 (2010).

${ }^{45}$ A. Santillán and S. I. Bozhevolnyi, Phys. Rev. B 84, 064304 (2011).

${ }^{46}$ F. J. García de Abajo, Rev. Mod. Phys. 79, 1267 (2007).

${ }^{47}$ K. Kambe, Z. Naturforsch. A 23, 1280 (1968).

${ }^{48}$ J. Christensen, L. Martín-Moreno, and F. J. García-Vidal, Appl. Phys. Lett. 97, 134106 (2010). 